\title{
94.8 Km-Range Direct Detection Fiber Optic Distributed Acoustic Sensor
}

\author{
Faruk Uyar $^{1}$, Talha Onat ${ }^{1}$, Canberk Unal ${ }^{1}$, Tolga Kartaloglu ${ }^{1}$, Ibrahim Ozdur ${ }^{1,2}$ and \\ Ekmel Ozbay ${ }^{1,3,4}$ \\ ${ }^{I}$ Nanotechnology Research Center, Bilkent University, Ankara, Turkey \\ ${ }^{2}$ Department of Electrical and Electronics Engineering, Abdullah Gul University, Kayseri, Turkey \\ ${ }^{3}$ Department of Electrical and Electronics Engineering, Bilkent University, Ankara, Turkey \\ ${ }^{4}$ Department of Physics, Bilkent University, Ankara, Turkey \\ faruk.uyar@bilkent.edu.tr
}

\begin{abstract}
This work demonstrates an ultra-long range direct detection fiber optic distributed acoustic sensor which can detect vibrations at a distance of $94.8 \mathrm{~km}$ with $10 \mathrm{~m}$ resolution along the sensing fiber. (C) 2019 The Author(s)

OCIS codes: (060.2370) Fiber optics sensors; (280.4788) Optical sensing and sensors; (120.4825) Optical time domain reflectometry.
\end{abstract}

\section{Introduction}

Phase-sensitive optical time domain reflectometry ( $\varphi$-OTDR) based distributed acoustic sensors (DAS) have been utilized as a powerful tool for real-time monitoring of oil and gas pipelines, intrusion detection, critical facility security, various vibration measurements and so on [1]. For linear assets monitoring, such as pipeline and national borderline security, ultra-long range distributed sensors are desired. To achieve this, various DAS technologies have been reported including coherent detection scheme, Raman optical amplification assistance and distributed hybrid amplification [2-4]. However, all these approaches generally add a significant increase in the cost and complexity of the system.

In this paper, we present an ultra-long range direct detection $\varphi$-OTDR sensor system with simple modifications to the structure. Vibration detection at the distance of $94.8 \mathrm{~km}$ with a spatial resolution of $10 \mathrm{~m}$ is achieved by using cascaded acousto-optic modulators and optimizing the system components. To the best our knowledge, it is the longest-range direct detection $\varphi$-OTDR system reported up to date.

\section{Experimental Set-up}

The experimental set-up of the direct detection $\varphi$-OTDR based DAS system is shown in Figure 1. A high-coherence $(<1 \mathrm{kHz}$ linewidth) laser is used as the light source and a booster erbium doped fiber amplifier (EDFA) is used for amplification. Dual cascaded acousto-optic modulators (AOM) are adopted for generating ultra-high extinction ratio (ER) interrogation pulses. The first AOM generates slightly longer optical pulses than the second one in order to make synchronization easier. The optical pulse width is dictated by the second AOM. The interrogation pulses are launched into the fiber via a high isolation circulator. The Rayleigh backscattered light from the sensing fiber is guided into the second EDFA via the same circulator. The width of the optical pulses was set to $100 \mathrm{~ns}$ with $1 \mathrm{~ms}$ interrogation period. The second EDFA is a low noise and high gain pre-amplifier which operates at maximum gain. Following the EDFA, a relatively broader pass band and a very narrow pass band optical bandpass filters (OBPF) are employed in a cascaded arrangement to effectively filter out the amplified spontaneous emission (ASE) noise of the pre-amplifier EDFA. The filtered optical signal is converted to electrical signal by a photodetector (PD), amplified by a transimpedance amplifier (TIA) and acquired by a data acquisition (DAQ). The signal is then processed offline with a PC.

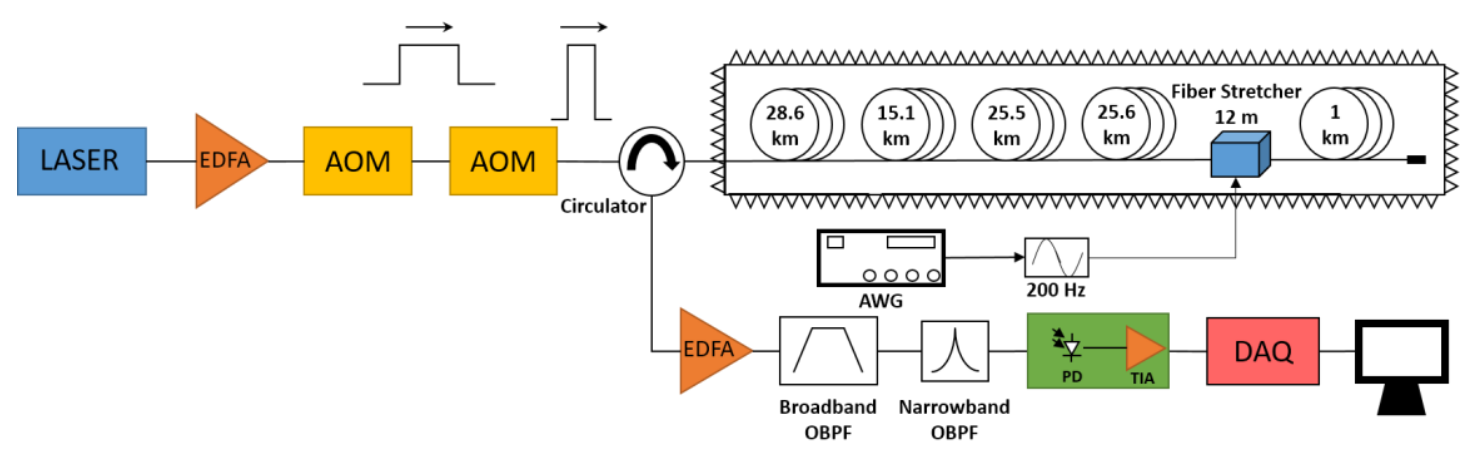

Fig. 1. Experimental set-up (Acronyms are explained in the text). 
One of the limiting factors of signal to noise ratio in $\varphi$-OTDR based DAS systems is the undesirable signal fluctuations caused by the leaked light due to the finite ER of the acousto-optic modulator (AOM). The AOMs employed in the experiment have ER values of $54 \mathrm{~dB}$ and $62 \mathrm{~dB}$. These values may seem more than enough for many applications, however, because of the very large duty cycle of the interrogation pulses, these values are not high enough to suppress the extinct light. The $\mathrm{CW}$ leaked light gets scattered from the fiber and results in a time variable background DC noise level at the photodetector.

The test fiber is placed in an isolated box for acoustic isolation. The test fiber is a $96 \mathrm{~km}$ long single-mode fiber (SMF-28), comprising fiber coils of $28.6 \mathrm{~km}, 15.1 \mathrm{~km}, 25.5 \mathrm{~km}, 25.6 \mathrm{~km}$ and $1 \mathrm{~km}$ connected together to obtain a $\sim 96$ $\mathrm{km}$ test fiber. A fiber stretcher is placed at the location of $94.8 \mathrm{~km}$ which generates vibration and induces phase shift at the specific section of the fiber. The stretcher is driven by an arbitrary waveform generator (AWG) which is set to generate $200 \mathrm{~Hz}$ sinusoidal wave with an amplitude of $4 \mathrm{~V}_{\mathrm{pp}}$.

\section{Results}

Coherent beating of the Rayleigh backscattered suppressed light results in a time varying noise. The effect of the AOM extinction ratio on the noise is analyzed by comparing the noise levels when the AOMs are completely turned off. Figure 2a shows the fluctuation noise for three different extinction ratio cases, $54 \mathrm{~dB}$ (AOM 1), $62 \mathrm{~dB}$ (AOM 2) and $>110 \mathrm{~dB}$ (AOM 1 and AOM 2 cascaded). It can be seen that the $54 \mathrm{~dB}$ ER modulator gives the largest noise as the leaked light power is the biggest, and the dual AOM case suppresses the noise below the noise floor.

The vibration detection performance of the system is measured at $94.8 \mathrm{~km}$ (corresponds to channel 9479) using the test fiber. The power spectral density of channel 9479 is shown in Figure $2 \mathrm{~b}$. The $200 \mathrm{~Hz}$ tone can be seen with an SNR value of $12.2 \mathrm{~dB}$.

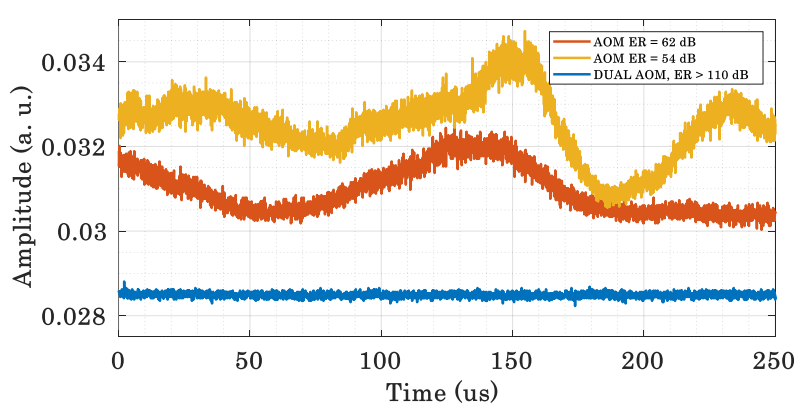

(a)

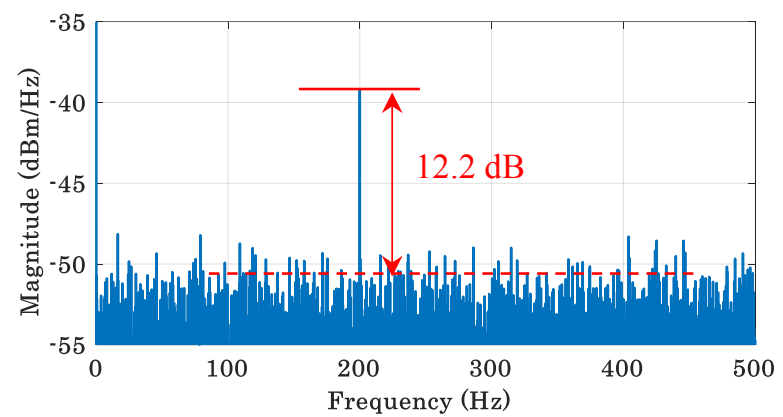

(b)

Fig. 2. a) Time domain channel data, b) Power spectral density of channel data.

The key design features to achieve these results are the usage of dual AOM in order to reduce the coherent noise caused by the finite ER and suppression of the ASE noise by the ultra-narrow pass bands optical filters. Also, the ultra-high sensitivity linear-gain photodetector and TIA units is another key component that contributes to range improvement.

\section{Conclusion}

In this work, a direct detection $\varphi$-OTDR based DAS system is presented which has $12.2 \mathrm{~dB}$ signal to noise ratio at the distance of $94.8 \mathrm{~km}$ with dual acousto-optic modulator architecture. To the best our knowledge, it is the longest-range reported direct detection $\varphi$-OTDR system. The use of dual AOM increases the extinction ratio of interrogation pulses to $>110 \mathrm{~dB}$. Due to the long range, the system has the potential to decrease the number of optical interrogators needed for long haul linear assets monitoring.

\section{References}

1. X. Liu, B. Jin, Q. Bai, Y. Wang, D. Wang, and Y. Wang, "Distributed fiber-optic sensors for vibration detection," Sensors 16, 1164 (2016).

2. L. Lv, F. Pang, H. Liu, X. Mei, J. Chen, Y. Li, T. Wang, Y. Song, "50-km-long distributed vibration fiber sensor based on phase-sensitive otdr using coherent detection," in Lasers and Electro-Optics Pacific Rim (CLEO-PR), 2017 Conference on, (IEEE, 2017), pp. 1-2.

3. F. Peng et al., H. Wu, X.-H. Jia, Y.-J. Rao, Z.-N. Wang, and Z.-P. Peng, "Ultra-long high-sensitivity $\varphi$-otdr for high spatial resolution intrusion detection of pipelines," Optics express 22, 13804-13810 (2014).

4. Z.Wang, J. Zeng, J. Li, M. Fan, H.Wu, F. Peng, L. Zhang, Y. Zhou, and Y. Rao, "Ultra-long phase-sensitive otdr with hybrid distributed amplification," Optics letters 39, 5866-5869 (2014). 